\title{
Propuesta metodológica para la evaluación de portales infomediarios de recursos abiertos en instituciones de educación superior
}

\section{Methodological Proposal for Evaluation of Open Source Portals Infomediaries in Higher Education Institutions}

\author{
Jenny Teresita Guerra González \\ Instituto de Investigaciones Bibliotecológicas e Información, \\ UNAM, México
}

Recibido: 09 de febrero 2016 Aceptado: 08 de abril 2016

Corregido: 15 de abril 2016 Publicado: 24 de junio 2016

\begin{abstract}
Resumen
El objetivo de este artículo consiste en proponer una metodología para evaluar cualitativamente el diseño, desarrollo e implementación de portales infomediarios para la distribución y difusión eficiente de recursos en acceso abierto en instituciones de educación superior. Lo anterior considerando que estos espacios académicos producen actualmente cantidades importantes de materiales digitales. A partir de una revisión bibliográfica sobre evaluación de sitios web e infomediación, así como de un análisis descriptivo de la información obtenida tras la revisión, se propuso la Metodología para la Evaluación de Portales Infomediarios de Recursos Abiertos en Instituciones de Educación Superior. La metodología incluye seis indicadores: contenido, comunidad, viralidad, servicios, necesidad y gestión del conocimiento. Cada indicador se califica con una serie de criterios que permiten verificar su cumplimiento mediante una escala de evaluación sumaria que los evalúa en eficiente, parcialmente eficiente o ineficiente.
\end{abstract}

\section{Palabras clave}

Portales web, Infomediarios, Recursos en acceso abierto, Distribución en línea, Difusión, Instituciones de Educación Superior, Evaluación.

\begin{abstract}
The objective of this article consisted in proposing a methodology to evaluate qualitatively the design, development and implementation of portals infomediaries for distribution and efficient dissemination of resource access in higher education institutions. This considering that these academic areas currently produce large quantities of digital material. From a literature review on evaluation web sites and infomediation, as well as a descriptive analysis of the information obtained after the review, set out the methodology for the assessment of infomediaries portals of open resources in institutions of higher education. The methodology includes six indicators: content, community, virality, services, need and knowledge management. Each indicator is qualified with a series of criteria that allow to verify its compliance through a summary evaluation that assesses them in efficient, partially efficient or inefficient scale.
\end{abstract}

\section{Keywords}

Web Portals, Infomediaries, Open Access Resources, Online Distribution, Diffusion, Higher Education Institutions, Evaluation.

\section{bibliotecas}




\section{Introducción}

La masificación e incorporación de las Tecnologías de la Información y la Comunicación (TIC) en las labores sustantivas de las instituciones de educación superior a nivel mundial, ha propiciado tanto el incremento de su producción académica y cultural como la distribución y difusión por Internet de esta clase de recursos. En ese tenor, universidades y otras instituciones promueven constantemente proyectos que facilitan el acceso a recursos de información propios y externos. Estas acciones se ligan al concepto de acceso y a las nociones de acceso a la información y disponibilidad de la información. Sobre el acceso, Torres (2010) señala que este posee dos acepciones: la primera al entenderlo como un proceso de aproximación a la información documental en el que se descubren aquellos materiales que probablemente contienen la información requerida y, en algunos casos, conocer dónde se localizan (catálogos, bases de datos). La segunda acepción se enfoca en la disponibilidad y el uso de la información. Existe disponibilidad cuando el documento está "físicamente" al alcance mientras que el uso se relaciona con la capacidad de leer y comprender por parte del sujeto.

Sánchez (2013) complementa lo anterior al distinguir entre acceso a la información y disponibilidad de la información aseverando que:

El acceso a la información en muchas ocasiones es confundido con la disponibilidad de la información. Se puede tener acceso a la información a través de instrumentos diversos como son los catálogos al público, los catálogos de publicaciones comerciales, las librerías, la world wide web y, por supuesto, a través de las bibliotecas. Tener acceso a la información no es solamente tener disponibilidad, sino también acceso a la información semánticamente relevante para ser interpretada por la comunidad, los grupos y las organizaciones de toda la sociedad. (p. 182-183)

Siguiendo esta línea considero que los recursos de información de las IES atraviesan, en primer lugar, un proceso de disponibilidad o existencia y posteriormente de acceso. McCreadie y Rice (1999) al concebir a la información como un bien o recurso que se puede producir, comprar, duplicar, distribuir, vender, comercializar, manipular o controlar abren la puerta para constituir y modelar entornos de información que comprenden propiedades sistémicas, maneras de interactuar, desarrollos internos y aplicaciones. Una vez establecidos los entornos de información o de recursos en nuestro caso, el siguiente paso es crear un sistema de acceso definido como las relaciones organizadas entre dos clases de sujetos; aquellos que asumen roles funcionales para buscar acceso a la información (usuarios) y aquellos que se comprometen con las actividades de adquisición, preservación, búsqueda, despliegue y presentación de los recursos conducentes hacia ese acceso (Schrader, 1992).

En ese tenor, el open access o acceso abierto (AA) es un sistema de acceso en el que las IES efectúan labores encaminadas a este. El despliegue y presentación de recursos como actividades de distribución y difusión en línea son aspectos de interés de este trabajo que toca tres aristas o temas: 1) el acceso abierto; 2) los portales infomediarios como herramientas integradoras de recursos dispersos de las IES que cuentan con múltiples plataformas y sitios de Internet y 3) la exposición de una metodología cualitativa para la evaluación de estos portales.

\section{bibliotecas}




\section{Los porqués del acceso abierto y su orientación actual}

Los comienzos formales del AA se ubican en las Declaraciones de Budapest (2002), Bethesda (2003) y Berlín (2003), siendo esta última la que resalta las amplias posibilidades que ofrece Internet en la difusión de cualquier tipo de conocimiento y según Abadal, Melero y Villaroya (2009: 159) se "establece con claridad cuáles son las dos formas para conseguir el acceso abierto: vía verde (el depósito de los documentos en repositorios) y la vía dorada (la publicación en revistas de acceso abierto)".

Sintetizando el espíritu de las tres declaraciones referidas, Suber (2006: 17) define al AA como "aquélla literatura digital, online, libre de pagos y libre de la mayoría de las restricciones que imponen el derecho de autor y las licencias". El mismo autor precisa, a la vez que desmitifica, el que este conlleve un acceso universal debido a que "aun cuando se haya alcanzado el AA, al menos cuatro tipo de barreras de acceso podrían seguir operando: filtros y censura; lenguaje; acceso y conectividad" (p. 28). De igual modo sostiene que el AA es compatible con el derecho de autor, la revisión por pares, los ingresos o incluso las ganancias, la impresión, la preservación, el prestigio, la catalogación, etc.

Aclaremos que el AA no se circunscribe a los materiales nacidos digitalmente, puede incluir aquellos impresos, filmes y demás documentos que han sido digitalizados. No se limita al texto sino que incorpora datos, audio, video, multimedia y códigos ejecutables (Suber, 20012). Es quizá por la denominada vía verde del acceso abierto por donde gran parte de estos recursos de información transitan.

La vía verde se asocia comúnmente a los repositorios institucionales y temáticos, pero en un sentido amplio engloba a toda clase de archivos digitales que sean diseminados por estos medios u otros existentes (portales web y plataformas) o que irán desarrollándose. La Organización de las Naciones Unidas para la Educación, la Ciencia y la Cultura (UNESCO, 2013: 22) publicó las Directrices para políticas de desarrollo y promoción del acceso abierto en las que afirma que "la ruta "verde" (...) puede capturar más material, más rápido, si se aplican políticas correctas". Una de las ventajas de la vía o ruta verde es que "todos los contenidos se adhieren a un mismo conjunto de reglas técnicas" (Rodríguez, 2014: s/p).

Así las cosas, el AA actúa como un modelo de distribución y difusión de recursos o contenidos en acceso abierto, que para Ramírez (2012) son entendidos como:

aquellos que permiten su consulta en línea de manera gratuita, sin restricciones técnicas o legales, todo ello con la finalidad de promover la difusión del conocimiento científico y el patrimonio cultural, así como incrementar la colaboración científica y académica, para generar nuevo conocimiento. El acceso abierto implica, además de la consulta de los materiales académicos, que se habilite su descarga, copia, distribución y/o impresión, es decir, todas aquellas acciones que permitan al usuario procesar los materiales para facilitar su manipulación, análisis y estudio. (p. 23)

\section{bibliotecas}


En ese sentido, Abadal, Melero y Villaroya (2009: 161) afirman que "cuando una institución quiere llevar a la práctica un programa de fomento del acceso abierto necesita convertir en acciones concretas aquellos instrumentos que haya elegido como más adecuados". Estos autores proponen seis mecanismos con sus correspondientes instrumentos que lo harían viable, expuestos en el cuadro 1.

Cuadro 1

Mecanismos e instrumentos para un programa institucional de fomento al acceso abierto

\begin{tabular}{ll}
\hline \multicolumn{1}{c}{ Mecanismos } & \multicolumn{1}{c}{ Instrumentos } \\
\hline Establecimiento de & $\checkmark$ Creación y mantenimiento de repositorios institucionales. \\
infraestructuras y prestación de & $\checkmark$ Edición de revistas en acceso abierto. \\
servicios & $\checkmark$ Creación de oficinas de asesoramiento en acceso abierto. \\
\hline \multirow{3}{*}{ Información y difusión } & $\checkmark$ Realización de campañas institucionales. \\
& $\checkmark$ Organización de congresos y conferencias. \\
& $\checkmark$ Elaboración de manifiestos. \\
\hline \multirow{2}{*}{ Incentivación económica } & $\checkmark$ Asignaciones presupuestarias a departamentos o grupos de investigación \\
& $\checkmark$ f función de objetivos de acceso abierto. \\
& $\checkmark$ Ayudas directas para el auto-archivo en repositorios. \\
& $\checkmark$ Subvención a la publicación en revistas de acceso abierto. \\
& institucional a portales en acceso abierto. \\
\hline Coordinación Institucional & $\checkmark$ Establecimiento de políticas y acciones conjuntas entre instituciones. \\
& $\checkmark$ Adhesión a declaraciones y manifiestos. \\
\hline \multirow{2}{*}{ Reglamentación } & $\checkmark$ Obligación de depositar resultados derivados de convocatorias propias. \\
& $\checkmark$ Obligación de depositar todas las publicaciones de los miembros de la \\
\hline Inspección y control & organización. \\
\hline
\end{tabular}

Fuente: Abadal, Melero y Villaroya (2009: p.161)

Se encuentra en los planteamientos de Abadal, Melero y Villaroya (2009) una carencia importante al no proponer instrumentos de inspección y control para fomentar el AA desde las instituciones. Sabemos, como ellos argumentan, que:

(...) una de las dificultades que comporta esta fase se refiere a los medios que se necesitarían para poder controlar de forma efectiva el establecimiento de mandatos. Se trata de un aspecto que muy pocas organizaciones se han planteado públicamente ya que de momento están centradas en establecer líneas de apoyo a la difusión u obligación. (p 167)

\section{bibliotecas}


También es necesario agregar como cuarto instrumento del mecanismo (establecimiento de infraestructuras y prestación de servicios) el desarrollo de portales o plataformas web. Lo anterior porque del 2012 a la fecha, estos han experimentado un uso masivo para la inserción, consulta y descarga de contenidos diversos que van de las aplicaciones (apps) y recursos de aprendizaje al software y el crowdsourcing de carácter público o privado.

Para Aguado (2013) el boom de los dispositivos móviles ha contribuido directamente con este fenómeno. A modo de ejemplo, el Onceavo estudio sobre los hábitos de los usuarios de Internet en México 2015 de la Asociación Mexicana de Internet (AMIPCI) encontró que de los 1662 encuestados, el 58\% acceden a Internet desde un Smartphone mientras que un 31\% lo hace por medio de una tableta electrónica. Navegar en Internet fue la segunda actividad realizada por los usuarios de teléfonos inteligentes; $82 \%$ de ellos descargaron al menos una app los meses previos a la aplicación del instrumento (abril de 2015).

\section{Portales Web}

Las características del entorno digital y sus múltiples productos han propiciado la aparición, desde comienzos de los años noventa, de nuevas formas de organización documental, distribución y difusión en línea. Como señalan Lafuente y Rosas (1998):

No hay que perder de vista que la publicación electrónica introduce la idea de la actualización parcial de un documento digital, sin necesidad de modificar, toda la estructura del mismo. Aunada a esta idea, se encuentra el fenómeno de la disponibilidad para su consulta, casi al mismo tiempo en que ésta se realiza (...). De tal manera, que nos encontramos frente a un producto editorial que tiene una serie de características que lo conforman como un producto acabado, pero que a diferencia de un impreso, este se puede actualizar fácilmente. (p. 188)

Los primeros esfuerzos de distribución y difusión de información y publicaciones electrónicas vía Internet fueron los portales web que en la última década del siglo XX tuvieron un importante auge. En aquel entonces portales de tipo horizontal, diseñados para atraer audiencias de toda clase y que ofrecían información muy variada (noticias, deportes, horóscopos, clima, etc.), como Yahoo!, $A O L$, Terra, entre otros, se posicionaron como proveedores de contenidos de actualidad a la par que espacios para la publicidad en el contexto de la web 1.0.

Si bien los portales horizontales continuaron ocupándose, su uso disminuyó en pro de los portales verticales. Estos son sitios web que proveen de información y servicios a un público en particular con contenidos específicos centrados en un tema. Sus ventajas consisten en la información de calidad centrada en el usuario, los anunciantes están en posibilidad de conocer de manera más eficiente los intereses de su mercado meta cuando el portal tiene un carácter comercial, entre otros. Tanto los portales verticales como los horizontales pueden beneficiarse con nuevos usuarios al adaptar su interfaz a la consulta en dispositivos móviles.

\section{bibliotecas}


La primera etapa de los portales web de las IES en el mundo (1995-2005) estuvo caracterizada por sitios de talante informativo que describían la historia y los servicios de la institución; eventos y noticias relacionados con la vida académica; direcciones de los centros de estudios; teléfonos y correos electrónicos de la planta académica, entre otros (Luna, Aguaded, Martínez y García, 2004). Aún no se explotaba la interactividad facilitada por Internet, quedando para más adelante procesos complejos como la interactividad comunicacional (comunicación en tiempo real) y la interactividad transaccional (procesos elaborados de enseñanza-aprendizaje; compras, etc.).

El portal web o portal de Internet definido como un sitio web a través del cual podemos acceder a multitud de recursos y de servicios, entre los que suelen encontrarse buscadores, foros, documentos, aplicaciones, compra electrónica, y otros, hace posible la interacción entre gestores e información y usuarios e información. Se sostiene sobre tres pilares sin importar su tipo: 1) información: buscadores, noticias, catálogos; 2) participación: email, chat, foros, redes sociales y 3) comodidad: en un solo espacio está todo a la mano (Ministerio de Educación, Cultura y Deportes de España, 2008; Portal Telepieza, 2013; Saorín, 2004). Así el diseño del portal web debe estar orientado a facilitar tanto la consulta como a obtener una idea acerca de la estructura, tamaño y forma de los contenidos que aloja.

En el entendido de que los portales son herramientas en la distribución y difusión de recursos digitales y diversas formas de publicación electrónica sustentadas en la idea de los servicios de información; los mecanismos de sistematización, acceso y disponibilidad son elementos fundamentales para evaluar la calidad de los contenidos. A ese respecto organizaciones e instituciones han propuesto indicadores y criterios; un ejemplo es el del Ministerio de Educación de Argentina (2013) que plantea las especificaciones consignadas en el Cuadro 2.

\section{Cuadro 2}

Variables para la evaluación de sitios web

\begin{tabular}{|c|c|c|}
\hline Indicador & Definición del criterio & Objetivos de la evaluación \\
\hline Autoridad & $\begin{array}{l}\text { La autoridad depende del responsable del sitio } \\
\text {-individuo, grupo, institución- y las fuentes } \\
\text { utilizadas. }\end{array}$ & $\begin{array}{l}\text { Que la información provenga de fuentes } \\
\text { confiables, específicas, actualizadas y reconocidas } \\
\text { en los ámbitos científicos y académicos. }\end{array}$ \\
\hline Actualización & $\begin{array}{l}\text { Laactualización puedereferirse ala incorporación } \\
\text { periódica de nuevos recursos o a la modificación } \\
\text { de los recursos y los datos existentes en respuesta } \\
\text { a la aparición de nuevos aportes al tema. }\end{array}$ & $\begin{array}{l}\text { Identificar sitios y recursos que contengan } \\
\text { información actualizada, si esto es pertinente } \\
\text { para su validez (por ejemplo: informaciones } \\
\text { periodísticas, científicas, tecnológicas, etc.). }\end{array}$ \\
\hline Navegabilidad & $\begin{array}{l}\text { Facilidad que se le ofrece al usuario de ubicarse } \\
\text { y moverse dentro del sitio. }\end{array}$ & $\begin{array}{l}\text { Seleccionar sitios que tengan una estructura } \\
\text { clara y ordenada de sus componentes y un diseño } \\
\text { que facilite la orientación del usuario durante } \\
\text { toda la navegación. Tomar en consideración que } \\
\text { en los portales web la navegación por parte del } \\
\text { usuario necesita ser intuitiva. }\end{array}$ \\
\hline
\end{tabular}

\section{bibliotecas}




\begin{tabular}{|c|c|c|}
\hline Indicador & Definición del criterio & Objetivos de la evaluación \\
\hline Organización & $\begin{array}{l}\text { Se espera que el sitio se encuentre ordenado } \\
\text { lógicamente y que cada segmento de } \\
\text { información se relacione con los demás. }\end{array}$ & $\begin{array}{l}\text { Seleccionar sitios que permitan encontrar con } \\
\text { facilidad y eficiencia la información en la forma } \\
\text { más autónoma posible. }\end{array}$ \\
\hline $\begin{array}{l}\text { Selección de } \\
\text { contenidos }\end{array}$ & $\begin{array}{l}\text { Se espera que los contenidos del sitio } \\
\text { manifiesten cuidado en el tratamiento y el } \\
\text { enfoque de sus temas. }\end{array}$ & $\begin{array}{l}\text { Identificar sitios y recursos que incluyan contenidos } \\
\text { válidos, consistentes, relevantes y significativos para } \\
\text { el nivel y las características de los destinatarios, y } \\
\text { que contemplen el uso apropiado del lenguaje y de } \\
\text { la comunicación escrita y gráfica. }\end{array}$ \\
\hline Legibilidad & $\begin{array}{l}\text { La legibilidad responde a una buena } \\
\text { combinación de colores, tamaños y tipos de } \\
\text { letras, fondos e ilustraciones, que permite } \\
\text { leer en la pantalla y navegar de una manera } \\
\text { adecuada y atractiva. }\end{array}$ & $\begin{array}{l}\text { Identificar sitios y recursos que sean de fácil lectura } \\
\text { y navegabilidad, donde los diferentes componentes } \\
\text { y recursos tipográficos e iconográficos añadan un } \\
\text { valor didáctico -cuando sea el caso- y estético a los } \\
\text { contenidos y no sean elementos distractores. }\end{array}$ \\
\hline $\begin{array}{l}\text { Adecuación al } \\
\text { destinatario }\end{array}$ & $\begin{array}{l}\text { Se entiende por destinatario al usuario al que } \\
\text { van dirigidos los contenidos y servicios del sitio } \\
\text { web (niños, docentes, familias, etc.). Puede ser } \\
\text { más o menos definido, más o menos amplio. }\end{array}$ & $\begin{array}{l}\text { Verificar que el contenido que se presenta sea } \\
\text { adecuado en nivel de complejidad, intereses, tipo } \\
\text { de estética, etc., al destinatario al que se dirige. }\end{array}$ \\
\hline
\end{tabular}

Fuente: Ministerio de Educación de Argentina (2013).

Los cuatro primeros indicadores son de fácil cumplimiento cuando se emplea un Content Managment System (CMS) porque este software permite implantar una estructura de soporte (framework) para la creación, edición, gestión, publicación y difusión de contenido digital multimedia en diversos formatos. El output de estos contenidos son páginas web dinámicas que interactúan con el servidor web para generarlas bajo petición del usuario con un formato predefinido, el contenido se extrae de la base de datos del servidor. Los restantes tres indicadores (selección de contenidos, legibilidad y adecuación al destinatario) requieren de la intervención humana directa porque atienden a cuestiones psicopedagógicas y didácticas.

\section{Los portales web como infomediarios}

Un infomediario es, siguiendo a Hagel y Singer (1999: 15), "un sitio web que recolecta y organiza cantidades grandes de datos, y actúa como intermediario entre los que desean la información y los que proveen la información". Este concepto puede incluir a los portales y a cualquier otro producto web en el que un agente funja como mediador para administrar información y proporcionar un servicio que satisfaga las necesidades requeridas por el usuario. Los infomediarios reúnen, organizan y vinculan la información con servicios agregados en línea. Son una buena alternativa al enorme reto de organizar grandes volúmenes de contenido académico que producen las universidades y sus comunidades (Peñaloza, 2015).

\section{bibliotecas}


Retomo aquí la concepción de los portales infomediarios como modelo de negocio de Internet para precisar algunas de las funciones que deben llevar a cabo, tengan o no un propósito comercial:

> Servir como depósito central de toda la información y datos relevantes solicitados por el usuario sintetizándolos, distribuyéndolos y preservándolos.

$>$ Proporcionar servicios de búsquedas complejas a través de su simplificación.

$>$ Garantizar la integridad de la información y los datos por medio de un análisis de su recepción aplicado a las distintas fuentes de origen.

$>$ Actuar como un ente neutral responsable de la recolección de la información, generando pronósticos de fondo y diseñando pautas para la creación de demanda.

En este trabajo, los portales web son entendidos como infomediarios de contenido, es decir, plataformas tecnológicas que facilitan su agregación, clasificación y distribución. El desarrollo de los portales web con este carácter fue previsto en los primeros años del siglo XXI (López y Martínez, 2002). Su evolución e importancia ha propiciado que de 2008 a la fecha se hable de un sector infomediario en países como España ligado directamente al reúso de datos (Hípola y Muñoz, 2015).

\section{Metodología y Resultados}

El objetivo general de este artículo es evaluar el uso de portales web infomediarios para la distribución y difusión eficiente de recursos en acceso abierto en Instituciones de Educación Superior de gran tamaño y productoras de cantidades importantes de materiales digitales, por lo que se procedió a:

a. Efectuar un proceso de revisión bibliográfica sobre evaluación de sitios web e infomediación para citar las bases teórico-metodológicas del caso de estudio.

b. Realizar un análisis descriptivo de la información obtenida tras la revisión bibliográfica. El análisis descriptivo consiste en especificar propiedades importantes de comunidades, grupos o cualquier otro fenómeno a estudiar. Un análisis de este tipo selecciona una serie de cuestiones y mide cada una de ellas independientemente para así describir los conceptos o variables que se investigan (Díaz, 2009).

De este modo se utilizaron dos herramientas cualitativas que permitieron obtener una serie de indicadores que debe cumplir un portal infomediario eficiente en IES, los cuales se sistematizan en la Metodología para la Evaluación de Portales Infomediarios de Recursos Abiertos en Instituciones de Educación Superior.

Con la aplicación de la primera herramienta se logró la definición de la variable infomediación, entendiendo por esta la condición ligada al tráfico de información de actualidad, datos o cifras que obliga a portales web, buscadores y comunidades virtuales a cumplir con una serie de condiciones para tornarse eficientes. Posteriormente, se eligieron seis de los ocho indicadores de los portales infomediarios comerciales propuestos por Rojo (2003) en su artículo El diseño del sitio web como herramienta estratégica de la imagen de marca. El autor menciona las condiciones (indicadores):

\section{bibliotecas}




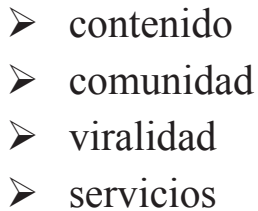

$>$ necesidad
$>$ gestión del conocimiento
$>$ comercio
$>$ matching

De acuerdo con el objetivo de la investigación se excluyeron las dos últimas, esto porque en relación con el AA en IES de carácter público, el primero es prescindible, mientras que el matching fue descartado ya que este es el conocimiento de los usuarios para generar transacciones entre grupos de ellos. Las transacciones en Internet comprenden actividades de negocios como compras, abastecimiento, ventas, transporte y almacenaje.

Una vez definidos los indicadores, se establecieron criterios para describirlos. Los criterios sintetizan las aportaciones de Rojo (2003), Góngora, Rodríguez, León, y Capote (2013) y Luna et al (2004). Para calificar el cumplimiento de estos y la concreción del indicador respectivo se propuso una escala de evaluación sumaria capaz de cualificar (vía la cuantificación) unidades de referencia concernientes a la eficiencia. Tales unidades son de tres tipos: eficiente, parcialmente eficiente e ineficiente. El Cuadro 3 condensa los seis indicadores, sus correspondientes criterios a evaluar en un portal infomediario y sus unidades de referencia para calificarlos. Es de forma detallada, la Metodología para la Evaluación de Portales Infomediarios de Recursos Abiertos en Instituciones de Educación Superior que se propone.

Cuadro 3

Metodología para la Evaluación de Portales Infomediarios de Recursos Abiertos en Instituciones de Educación Superior

Indicador

1) Contenido: Información útil, variada y actual.
Criterios

1.1 Existe un plan de difusión y distribución de recursos a corto, mediano y largo plazo.

1.2 La elección de los recursos a difundir y distribuir es congruente con la misión de la IES, plan de desarrollo institucional, líneas de investigación y/o docencia, mandatos institucionales o nacionales de AA, etc.

1.3 Se definen claramente los tipos de contenidos o recursos que se integrarán al portal así como su procedencia (agregadores internos, agregadores externos/ modelo híbrido).

1.4 Los formatos de los recursos emplean software libre para su consulta.

1.5 El diseño del sitio y desarrollo de la interfaz obedecen a un proceso de arquitectura de la información.

1.6 El volumen de información por categoría, campo o materia es homogéneo.
Calificación hacia la eficiencia

Eficiente:

sí los seis criterios se cumplen

Parcialmente eficiente:

sí se cumplen cuatro de los seis criterios

Ineficiente:

si solo se cumplen tres o menos criterios

\section{bibliotecas}




\begin{tabular}{|c|c|c|c|c|}
\hline \multicolumn{2}{|r|}{ Indicador } & \multicolumn{2}{|r|}{ Criterios } & \multirow[t]{2}{*}{ Calificación hacia la eficiencia } \\
\hline \multirow[t]{5}{*}{ 2) } & \multirow{5}{*}{$\begin{array}{l}\text { Comunidad: Nexo de unión } \\
\text { entre todos los participantes } \\
\text { basado en intereses, } \\
\text { afinidades, ideología u otros } \\
\text { valores. }\end{array}$} & 2.1 & El portal tiene presencia en redes sociales. & \\
\hline & & 2.2 & Las redes sociales informan oportunamente & \multirow{4}{*}{$\begin{array}{l}\text { Eficiente: } \\
\text { si los cuatro criterios se cumplen } \\
\text { Parcialmente eficiente: } \\
\text { si se cumplen entre tres y dos de } \\
\text { los criterios } \\
\text { Ineficiente: } \\
\text { si solo se cumplen un criterio o } \\
\text { ninguno }\end{array}$} \\
\hline & & & $\begin{array}{l}\text { de la existencia de contenidos o recursos } \\
\text { nuevos y redireccionan al portal. }\end{array}$ & \\
\hline & & 2.3 & $\begin{array}{l}\text { Existe interconexión interna del portal con } \\
\text { otros sitios web o plataformas de la IES. }\end{array}$ & \\
\hline & & 2.4 & $\begin{array}{l}\text { Existe interconexión externa del portal con } \\
\text { otros sitios web o plataformas de interés para la } \\
\text { IES a través de alianzas, de tal modo que llega a } \\
\text { usuarios no considerados en un principio. }\end{array}$ & \\
\hline \multirow[t]{3}{*}{ 3) } & \multirow[t]{3}{*}{$\begin{array}{l}\text { Viralidad: Modelo de } \\
\text { diseminación a gran escala, } \\
\text { rápida y económica. }\end{array}$} & 3.1 & $\begin{array}{l}\text { Creación y divulgación de una guía o manual } \\
\text { institucional para la administración de redes } \\
\text { sociales y cuentas institucionales. }\end{array}$ & \multirow{3}{*}{$\begin{array}{l}\text { Eficiente: } \\
\text { si los tres criterios se cumplen } \\
\text { Parcialmente eficiente: } \\
\text { si se cumplen dos criterios } \\
\text { Ineficiente: } \\
\text { si solo se cumple un criterio o } \\
\text { ninguno }\end{array}$} \\
\hline & & 3.2 & $\begin{array}{l}\text { Se establecen parámetros para medir la viralidad } \\
\text { del portal y las redes sociales del portal. }\end{array}$ & \\
\hline & & 3.3 & $\begin{array}{l}\text { Se emplea software especializado en gestión } \\
\text { de la comunicación. }\end{array}$ & \\
\hline \multirow[t]{6}{*}{ 4) } & \multirow[t]{6}{*}{$\begin{array}{l}\text { Servicios: Adaptación al tipo } \\
\text { de usuario o transacción. }\end{array}$} & 4.1 & $\begin{array}{l}\text { El portal cumple con características de } \\
\text { comunicabilidad, usabilidad y accesibilidad. }\end{array}$ & \multirow{6}{*}{$\begin{array}{l}\text { Eficiente: } \\
\text { si los seis criterios se cumplen } \\
\text { Parcialmente eficiente: } \\
\text { si se cumplen cuatro de los seis } \\
\text { criterios } \\
\text { Ineficiente: } \\
\text { si solo se cumplen tres o menos } \\
\text { criterios }\end{array}$} \\
\hline & & 4.2 & $\begin{array}{l}\text { Todos los contenidos disponibles en el portal } \\
\text { están en AA y no presentan ninguna condición } \\
\text { (software, password,) para su consulta y uso. }\end{array}$ & \\
\hline & & 4.3 & $\begin{array}{l}\text { Se alienta al reúso y conversión de los recursos } \\
\text { a través de licencias. }\end{array}$ & \\
\hline & & 4.4 & $\begin{array}{l}\text { El portal cuenta con un motor de búsqueda que } \\
\text { permite la búsqueda simple y especializada. }\end{array}$ & \\
\hline & & 4.5 & $\begin{array}{l}\text { El portal integra sus redes sociales a la } \\
\text { interfaz. }\end{array}$ & \\
\hline & & 4.6 & $\begin{array}{l}\text { El portal cuenta con servicios de streaming, } \\
\text { webcast, etc. }\end{array}$ & \\
\hline \multirow[t]{2}{*}{ 5) } & \multirow[t]{2}{*}{$\begin{array}{l}\text { Necesidad: Propiciar que el } \\
\text { público tenga que retornar } \\
\text { frecuentemente al sitio. }\end{array}$} & 5.1 & $\begin{array}{l}\text { La descripción de los recursos y el diseño de } \\
\text { la interfaz facilitan su disponibilidad, uso y } \\
\text { compartición. }\end{array}$ & \multirow{2}{*}{$\begin{array}{l}\text { Eficiente: } \\
\text { si los dos criterios se cumplen } \\
\text { Ineficiente: } \\
\text { si solo se cumple un criterio o } \\
\text { ninguno }\end{array}$} \\
\hline & & 5.2 & $\begin{array}{l}\text { La IES lleva a cabo campañas de uso de } \\
\text { los recursos alojados en el portal con su } \\
\text { comunidad y público externo. }\end{array}$ & \\
\hline
\end{tabular}




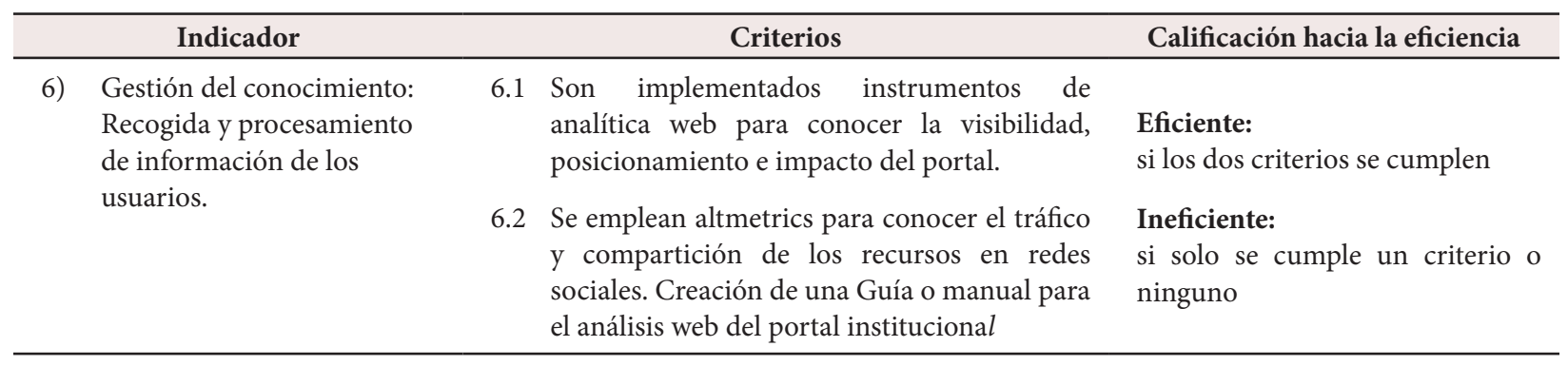

Fuente: Elaboración propia de la autora.

\section{Discusión}

Si tomamos en cuenta que los portales web verticales o especializados demandan la lealtad del usuario para considerarse exitosos, sus estrategias deberán dirigirse a incrementar el volumen y el valor de su información además de la actualización constante de sus contenidos. Los responsables del portal han de conocer el perfil de los usuarios y visitantes del sitio con el fin de orientarlos de manera adecuada.

El contenido es el factor clave que determina la presencia de un mayor número de usuarios en un portal infomediario. Este puede crearse desde cero o adaptarse de otros agregadores. Entre las buenas prácticas que necesitan implementar las instituciones de educación superior con respecto a sus portales están: promover una cultura de mejora continua de su contenido que incluya asegurar que cada servicio contenga metadatos adecuados - hay mayores probabilidades de éxito cuando se adopta un estándar de metadatos interoperables como Dublin Core-; validar y verificar todos los registros de catálogos de fichas en los repositorios y servicios relacionados. Para el caso de los Recursos Educativos Abiertos (REA) es indispensable una clasificación curricular que facilite su localización y uso por docentes y estudiantes (Peñaloza, 2015).

Las alianzas o vínculos con portales o sitios web externos son sumamente relevantes, pero antes de establecerlas es necesario evaluar su fiabilidad e identificar sí aportan valor añadido a nuestro portal. El sitio web asociado debe potenciar el contenido del portal y la atracción de usuarios. Las IES pueden realizar vínculos con asociaciones universitarias nacionales e internacionales, medios de comunicación locales, centros de investigación, sitios académicos y educativos, asociaciones de expertos, entre otras para tener presencia en sus plataformas de manera que desde ellas y otras herramientas complementarias, como redes sociales institucionales, se referencie a su portal.

Como destaca Peñaloza (2015: 9) "ampliar la diseminación de las contribuciones de las universidades vía Internet ha facilitado la comparación de su producción académica y científica en la arena internacional (...) no sólo en sus comunidades locales sino a nivel mundial". El agregado de este fenómeno es que estudiantes se están iniciando en la publicación de productos académicos de manera similar a como lo hacen los investigadores mientras que los profesores difunden sus productos académicos y el material didáctico que generan.

\section{bibliotecas}




\section{Conclusiones}

Las universidades a nivel internacional se han decantado por el uso de repositorios y páginas web para la diseminación de sus contenidos digitales en AA. Estos instrumentos son funcionales cuando el flujo de información no es tan grande. El aspecto de integración que brindan los portales va en dos sentidos: que las instituciones de educación superior aprovechen las facilidades que brindan los CMS u otros softwares para la distribución y difusión de contenidos, y que los mandatos o normativas sobre AA sean lo más completas posibles, de tal forma que el mayor número de recursos estén disponibles para su incorporación al portal web.

Además, lo que planteo es que la organización de los contenidos en los portales web vaya en consonancia con la razón de ser de la institución (misión, visión, estructura organizacional, plan rector) y sus líneas de trabajo (docencia, investigación, extensión de la cultura, entre otros). La meta es que el usuario vea los materiales digitales que produce la universidad representado lo más fiel posible en el portal de una forma sistemática acorde a una interfaz sencilla y amigable. La metodología aquí presentada contribuye tanto a la evaluación como a la mejora continua de este tipo de plataformas, esenciales para la educación superior actual.

\section{Referencias}

Abadal, E. (2001). Sistemas y servicios de información digital. Gijón: Ediciones Trea/Edicions de la Universitat de Barcelona.

Abadal, E., Melero, R. y Villaroya, A. Políticas institucionales para el fomento del acceso abierto: tipología y buenas prácticas. Bollettino aib, núm. 2, junio de 2009, pp. 159-170. [Artículo en línea] Disponible en: http://digital.csic.es/bitstream/10261/114808/1/2009-bollettino-abadal.pdf

Aguado, J. La industria del contenido en la era Post-Pc: Horizontes, amenazas y oportunidades. Pp. 5-31 en Canavilhas, J. coord. (2013). Notícias e Mobilidadeo jornalismo, na era dos dispositivos móveis. Covilhã: Universidade da Beira Interior (UBI)/Laboratório de Comunicação e Conteúdos Online.

Díaz, F. (2009). Metodología de la investigación científica y bioestadística. Santiago de Chile: Universidad Finis Terrae.

Directrices para políticas de desarrollo y promoción del acceso abierto (2013). París: UNESCO. [Artículo en línea] Disponible en: http://www.unesco.org/new/fileadmin/MULTIMEDIA/HQ/ $\mathrm{CI} / \mathrm{CI} / \mathrm{pdf} /$ publications/policy guidelines oa sp reduced.pdf

\section{bibliotecas}


Góngora, A., Rodríguez, A., León, Y., y Capote, T. (2013). Guía para evaluar Usabilidad, Accesibilidad y Comunicabilidad en Aplicaciones Web con Intervención del Usuario. Revista Antioqueña de las Ciencias Computacionales y la Ingeniería de Software, núm. 2, julio/diciembre, pp. 59-64. [Artículo en línea] Disponible en: http://fundacioniai.org/raccis/v3n2/n5a7.pdf

Hagel, J y Singer, M. (1999). Net Worth. Shaping Markets when Customers Make the Rules. Boston: Harvard Business School Press.

Hípola, P. y Muñoz, A. Evolución del mercado de la información electrónica: desde los orígenes hasta el auge del sector infomediario. Revista Española de Documentación Científica, núm. 2, abril/junio 2015, pp. 1-16.

Lafuente, R. y Rosas, A. La publicación electrónica: ¿Un paradigma de organización documental digital? Investigación Bibliotecológica, núm. 25, julio/diciembre de 1998, México, pp. 164-196.

López, R. y Martínez, F. La importancia de los productos documentales dentro de la gestión de los e-contents en los portales periodísticos. Ponencia presentada en la Conferencia Contenidos y Aspectos Legales en la Sociedad de la Información (CALSI), Valencia (España), 22-23 octubre 2002. [Artículo en línea] Disponible en: http://eprints.rclis.org/handle/10760/4311

Luna, P., Aguaded, J., Martínez, F., García, M. Hacia una metodología de análisis de sitios web universitarios. Ponencia presentada en EDUTEC 2004, Barcelona (España), 17-19 noviembre 2004. [Ponencia en línea] Disponible en: http://www.lmi.ub.es/edutec2004/pdf/902.pdf

McCreadie, M. y Rice, R. Trends in analyzing Access to information. Part I: cross-disciplinary conceptualization of access. Information Processing \& Management, núm.1, 1999, pp. 45-76.

Ministerio de Educación de la República Argentina (2013). Cómo evaluar sitios web. Disponible en: http://www.educ.ar/dinamico/UnidadHtml get 5f224bbd-7a0a-11e1-813e-15e3c494af/ index.html

Ministerio de Educación, Cultura y Deportes de España (2013). Concepto de portal web. Instituto Nacional de Tecnologías Educativas y de Formación del Profesorado. Disponible en: http:// roble.pntic.mec.es/jprp0006/tecnologia/bachillerato tic/unidad02 buscadores/buscadores04 portales.html

Onceavo Estudio sobre los hábitos de los usuarios de Internet en México 2015 (2015). México: Asociación Mexicana de Internet. [Material en línea] Disponible en: https://amipci.org.mx/images/ AMIPCI HABITOS DEL INTERNAUTA MEXICANO 2015.pdf

Peñaloza, M. OER strategies and best practices as success factors in Open Access initiatives in higher education. Ponencia presentada en la Open Education Global Conference 2015. Alberta, Canadá, 22 de abril de 2015. [Ponencia en línea] Disponible en http://conference.oeconsortium.org/2015/ wpcontent/uploads/2015/03/OEGlobal2015 MPB submission95 final.pdf

\section{bibliotecas}


Portal Telepieza (2008). Los diferentes tipos de portales que existen en Internet. [Artículo en línea] Disponible en: http://www.telepieza.com/wordpress/2008/01/29/los-diferentes-tipos-deportales-que-existen-en-internet

Ramírez, L. M. Contenidos de Acceso Abierto. Revista Digital Universitaria, núm. 4, abril., 2012. [Artículo en línea] Disponible en: http://www.revista.unam.mx/vol.13/num4/art46/art46.pdf

Rodríguez, A. Entre acuerdos y desacuerdos sobre las rutas de acceso a las colecciones digitales. Ponencia presentada en el $2^{\circ}$ Congreso Nacional de Bibliotecas en Ciencias dela Salud. 20 y 21 de noviembre de 2014. Instituto Nacional de Rehabilitación. México, D.F. [Ponencia en línea] Disponible en: http://www.bibliotecarios.concepto28.com/programa/entre-acuerdos-y-desacuerdos.pdf

Rojo, P. El diseño del sitio web como herramienta estratégica de la imagen de marca. Quaderns digitalis, febrero 2003. [Artículo en línea] Disponible en: http://www.quadernsdigitals.net/index. php?accionMenu=hemeroteca.VisualizaArticuloIU.visualiza\&articulo id $=5885$

Sánchez,E.J. Acceso a la información gubernamental: estudios y tendencias. Investigación Bibliotecológica, núm. 60, mayo / agosto, 2013, México, pp. 181-201.

Saorín, T. (2003). Los portales bibliotecarios. Madrid: Arco Libros.

Schrader, A. A system theory of access, en Pertti Vakkari y Blaise Cronin, Conceptions of library and information science: historical, empirical and theoretical perspectives, Taylor Graham, 1992, pp. 187-200.

Suber, P. (2006). Una introducción al acceso abierto en Babini, D. y Fraga, J. CLACSO, Consejo Latinoamericano de Ciencias Sociales. Buenos Aires. Pp 15-33. [Capítulo de libro en línea] Disponible en: http://bibliotecavirtual.clacso.org.ar/ar/libros/secret/babini/Peter\%20Suber.pdf

Suber, P. (2012). Open Access. Cambridge: The MIT Press.

Torres, G. A. (2010). El acceso universal a la información. Del modelo librario al digital. México: UNAM.

\section{Notas sobre la autora}

* Jenny Teresita Guerra González. Instituto de Investigaciones Bibliotecológicas y de la Información, Universidad Nacional Autónoma de México. Correo electrónico: jenny@iibi.unam.mx

\section{bibliotecas}

